\title{
Assessment of air pollutant PM2.5 pulmonary exposure using a 3D lung-on-chip model
}

Cong $X u^{a, d \#}$, Min Zhang ${ }^{a, d \#}$, Wenwen Chen $^{a, d}$, Lei Jiang ${ }^{a}$, Chunying Chen ${ }^{e}$, and Jianhua $\operatorname{Qin}^{a, b, c, d *}$

\section{AUTHOR ADDRESS}

a Division of Biotechnology, CAS Key Laboratory of Separation Sciences for Analytical Chemistry, Dalian Institute of Chemical Physics, Chinese Academy of Sciences, Dalian, China.

b Institute for Stem Cell and Regeneration, Chinese Academy of Sciences, Beijing, China.

c CAS Center for Excellence in Brain Science and Intelligence Technology, Chinese Academy of Sciences, Shanghai, China.

d University of Chinese Academy of Sciences, Beijing, China.

e CAS Key Laboratory for Biomedical Effects of Nanomaterials and Nanosafety and CAS Center for Excellence in Nanoscience, National Center for Nanoscience and Technology, Beijing, China \#These authors contributing equally to this work.

*Correspondence should be addressed to Jianhua Qin(jhqin@dicp.ac.cn). 


\section{Supporting Information}

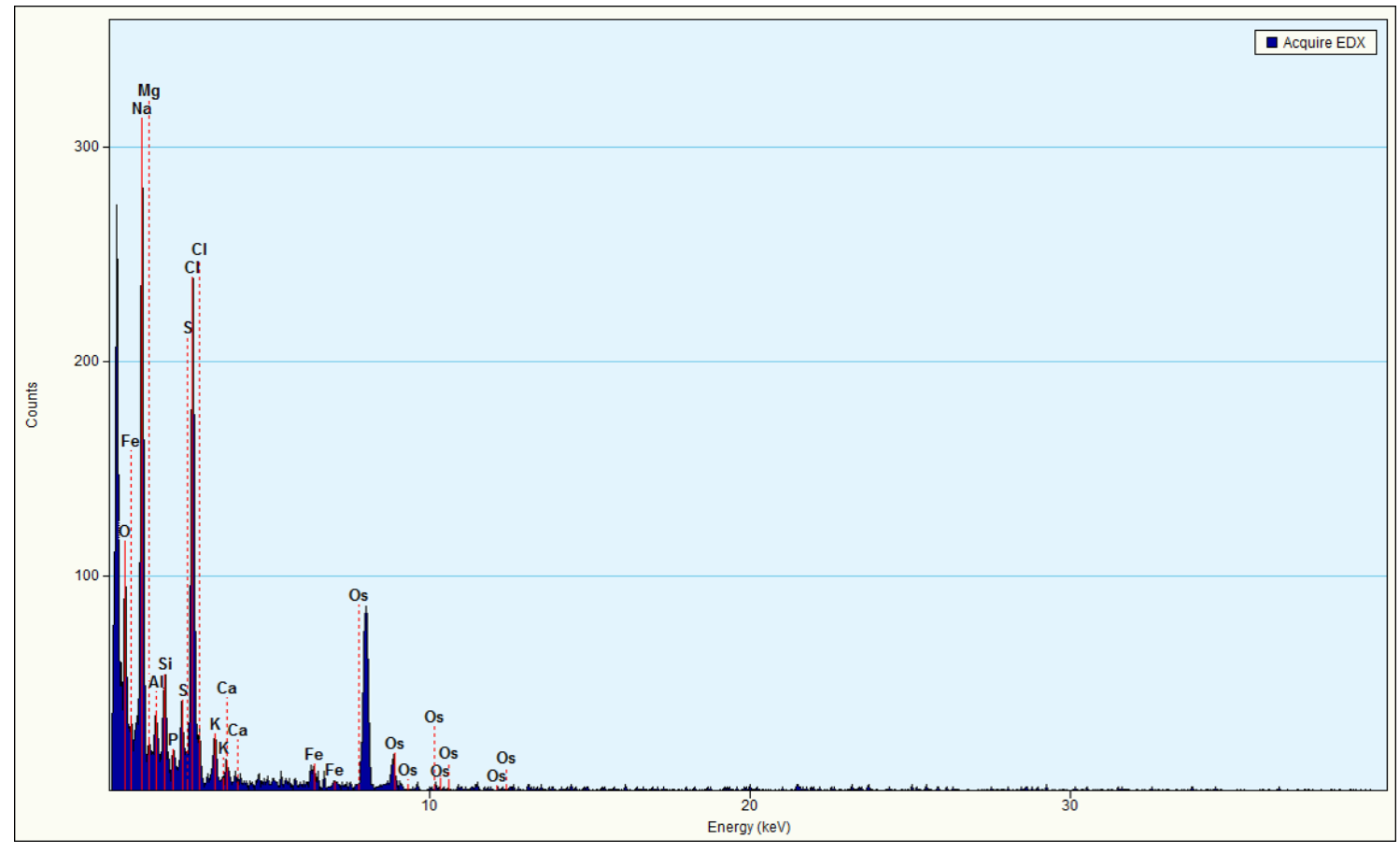

Figure S1. Elemental components of PM2.5 

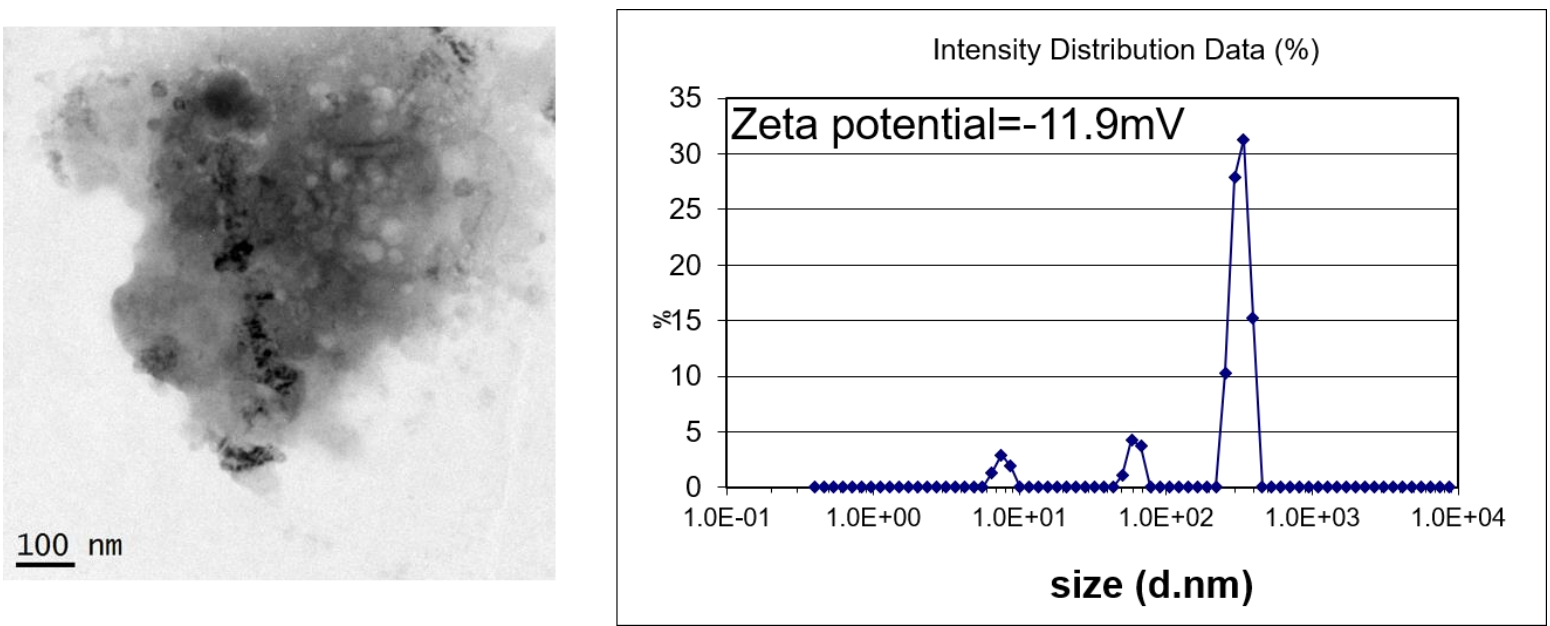

Figure S2. Left: A TEM image of PM2.5 aggregates derived from culture medium; Right: Zeta potential and size distribution of PM2.5 aggregates in culture medium.
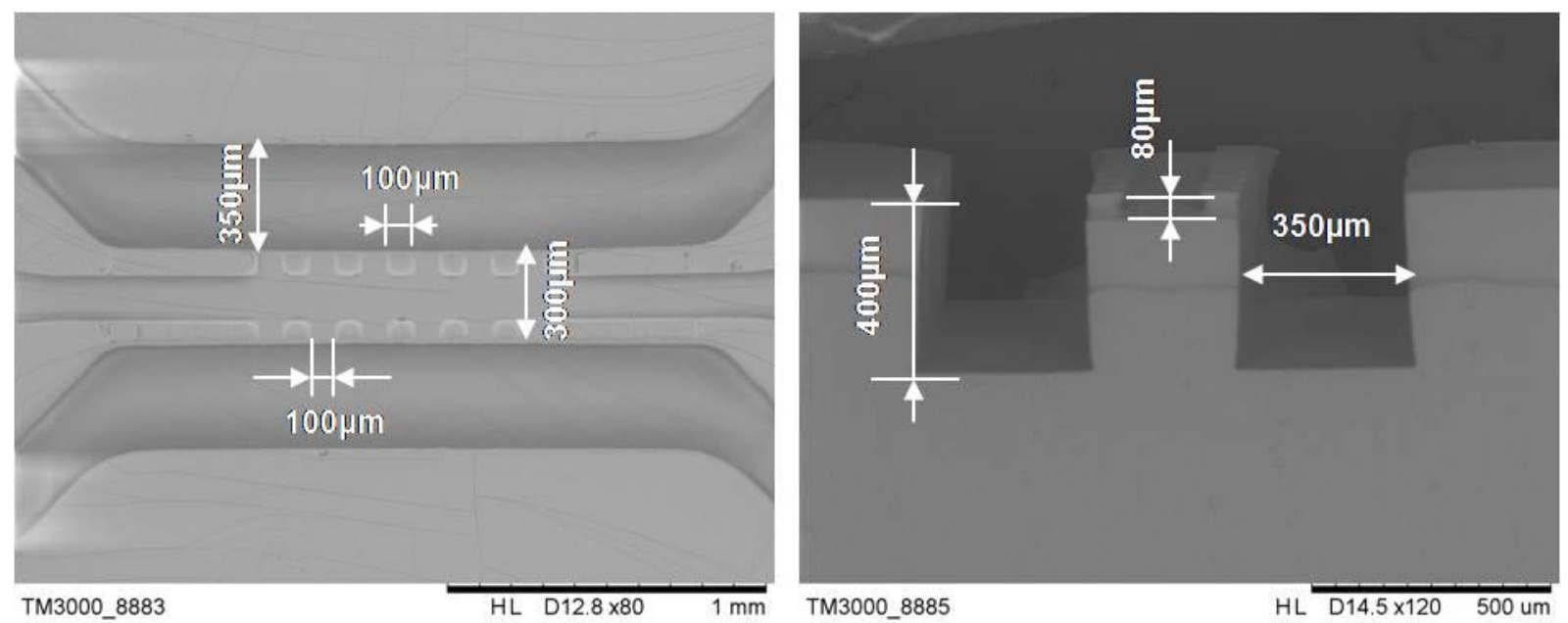

Figure S3. SEM images of the lung-on-a-chip. 


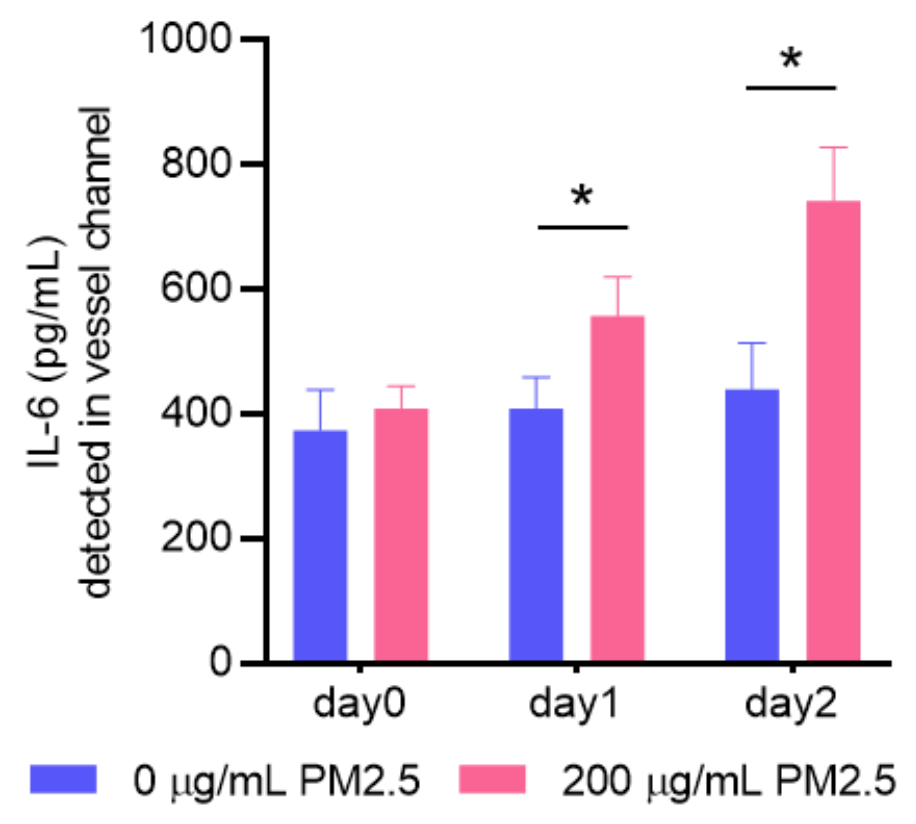

Figure S4. Increased IL-6 concentrations in culture media from vessel channels after 200 $\mu \mathrm{g} / \mathrm{mL}$ PM2.5 exposure for 3 days. * indicates $\mathrm{p}<0.05$; Error bar: $S \mathrm{D} ; \mathrm{n}=5$. 


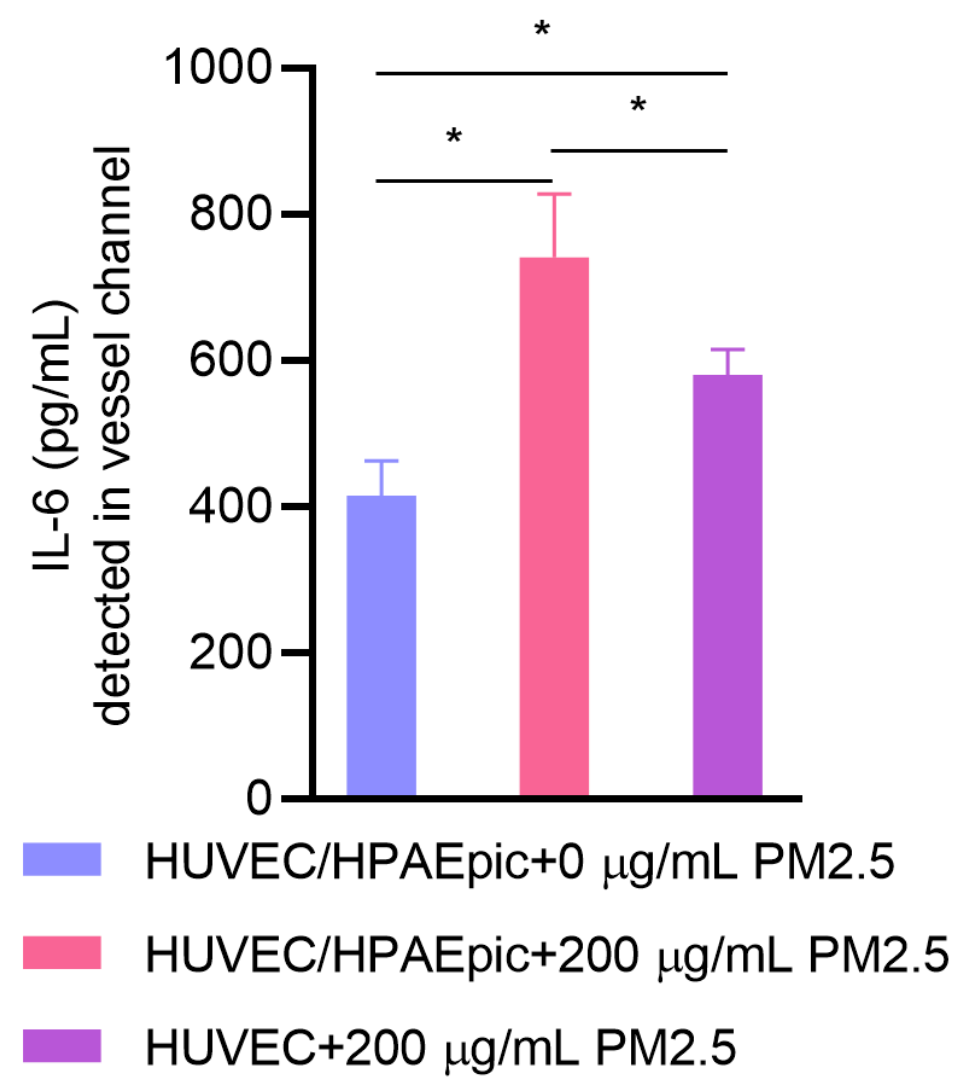

Figure S5. Increased IL-6 concentrations in culture media from vessel channels with coculture of HUVECs and HPAEpics than monoculture of HUVEC on the chip. $200 \mu \mathrm{g} / \mathrm{mL}$ PM2.5 treatment applied for three days. $n=5,{ }^{*}$ indicates $P<0.05$. 